\title{
Healthy Pork Production through Dietary n6:n3 Ratio Regulation
}

\author{
Jyun-Ru Yang ${ }^{1} \&$ Tu-Fa Lien ${ }^{1}$ \\ ${ }^{1}$ Department of Animal Science, National Chiayi University, Chiayi, Taiwan, R. O. C. \\ Correspondence: Tu-Fa Lien, Department of Animal Science, National Chiayi University, 300 University Road, \\ Luh Liau Li, Chiayi, Taiwan, R. O. C. Tel: 886-5-271-7536. E-mail: tflien@mail.ncyu.edu.tw
}

$\begin{array}{ll}\text { Received: September 15, } 2015 & \text { Accepted: November 2, } 2015 \quad \text { Online Published: December 15, } 2015 \\ \text { doi:10.5539/jas.v8n1p25 } & \text { URL: http://dx.doi.org/10.5539/jas.v8n1p25 }\end{array}$

\begin{abstract}
The meat fat fatty acid composition could influent consumer health. Thus, this study was though dietary n-6:n-3 ratio regulation to production healthy pork. The experiment was used eighty LYD pigs that average weight was $66.5 \mathrm{~kg}$ (half male and half female) divide into five groups, they are lard group (L), soybean oil group (SO), commercial fish product group (CFP), canola oil group (CO), and $50 \%$ fish oil and $50 \%$ canola oil group (FCO) with 4 replicates, this experiment was lasted for 90 days. Experimental results indicated that the growth performances was no difference among groups; serum cholesterol, LDL and LDL-C were lower, meanwhile HDL was higher $(\mathrm{P}<0.05)$ in FCO group than in control group. Back fat thickness, pork color, water holding capacity and meat fat content show no difference among groups. TBARS test on pork storage for 15 days in SO group was significantly higher $(\mathrm{P}<0.05)$ than $\mathrm{CO}$ group. The $\mathrm{n}-6: \mathrm{n}-3$ ratio of back and belly fat in CFP, FCO and $\mathrm{CO}$ groups were significantly lower $(\mathrm{P}<0.05)$ than in lard group. Panel evaluation score in SO group was significantly better $(\mathrm{P}<0.05)$ than $\mathrm{CO}$ group in flavor, texture, juicy and total acceptance in longissmus muscle, but no difference in belly meat. In conclusion, the pork n-6: n-3 ratio was decreased with CFP, FCO, and CO supplementation, feeding pigs with low n-6:n-3 ratio fat could production healthy pork for consumers.
\end{abstract}

Keywords: dietary fat, growth performance, carcass, fatty acid composition, n-6:n-3 ratio, pigs

\section{Introduction}

Meat products are important sources of dietary fat. The meat fat content and the fatty acid composition could deeply influent consumer health. In ordinary food, the much saturated fatty acid it contains, the better taste it does (Cameron \& Enser, 1991), but saturated fatty acid is highly corrected with artery and heart disease (Enser, 2001).

Previous reports showed that consumption of n-3 polyunsaturated fatty acid (n-3 PUFA) significantly reduced plasma triacylglycerol, cholesterol and blood pressure (Jiang \& Sim, 1992; Oh et al., 1994; Baik et al., 2010), and decrease atherogenic oxidative stress in vivo (Casós et al., 2010). In addition, Wan et al. (2010) indicated that the aortic lesion area was significantly reduced with lower ratio of n-6:n-3 fatty acids. A significant reduction of interleukin 6 and prostaglandin E-2 in both plasma and aorta culture medium was observed, this findings demonstrate that a decreased n-6:n-3 fatty acid ratio reduces atherosclerotic lesions in apoE(-/-) mice. This protective effect may be attributed to the antiinflammatory properties of n-3 fatty acids. The n-3 PUFA up-regulate several inflammation molecules including serum amyloid A (SAA), tumor necrosis factor-alpha (TNF-alpha) and interleukin-6 (IL-6) in hepatocytes and adipocytes. Actions of these inflammation mediators resemble those of n-3 PUFA in the modulation of many lipid metabolism-related genes (Tai \& Ding, 2010). Among the fatty acids, the n-3 PUFA is of especially importance for infant and elder, because they have limited capacity to elongate and desaturation during fatty synthesis (Simopoulos \& Salem, 1992).

Thus, lower ratio of n-6/n-3 PUFA meat products also good for consumer health. The Department of Health (UK) (1994) suggested that the ratio of polyunsaturated fatty acid: saturated fatty acid (P:S) should be higher than 0.4 and $n-6 / n-3$ ratio should be under 4 .

Commonly, the P:S ratios in pork could reach 0.58 , but the $n-6: n-3$ is too high, and is necessary to be reduced. The factors that affected meat fatty acids composition including breed, diet and type of meat (Wood et al., 2004), among them, the dietary fat is the most effective factor. Because the body fatty acid composition is very sensitive to the dietary fat, for instance, linseed (Kouba et al., 2008) and fish oil supplementation diet (García-Rebollar et al., 2008) could significantly increase n-3 PUFA content, and thus reduced animal body fat $n-6: n-3$ ratio. It is 
worth expecting, by using high PUFA and n-3 fatty acid rich feedstuffs to produce healthful meat via modifying meat fatty acid profile.

Fatty acid compositions would influence meat quality, such as firmness, color, antioxidant characters, flavor, tenderness and juiciness. The antioxidant capacity of fatty acid would influence the color of pork. For example, PUFA are easy oxidation and consequently, red oxymyoglobin change its color to brown metmyoglobin (Mottram, 1998).

The corn-soybean always is the basal diet of animals, corn contains 3-5\% of fat, the fatty acid composition are C18:1 (45.6\%) and C18:2 (45\%), no C18:3; in addition, the supplemental lipid most is used soybean oil if the cost is low, soybean oil contains C18:1 (17\%), C18:2 (54.4\%) and C18:3 (7.1\%), the $\mathrm{n}-6: \mathrm{n}-3$ ratio is 7.7 , it is higher than the expect value of 4 . Thus, it is need to seek for other dietary fat source to reduce the pork n-6:n-3 ratio.

Among all kind of lipid resources, the most abound n-3 PUFA are canola oil and fish oil; cod liver oil contains C18:2 (1.2\%), C20:5 (EPA, 10.9\%), C22:6 (DHA, 10.3\%), the $\mathrm{n}-6: \mathrm{n}-3$ ratio is 0.06 , but it is expensive, and may affect meat quality if adding at a high level. Canola oil contains C18:3 (9-11\%), C18:2 (13\%), n-6:n-3 ratio is 1.44 , which is the most lower ratio in plant lipid sources. Thus, in this study we selected contains linseed product, canola oil and/or plus fish oil to reduce the pork n-6:n-3 ratio.

The purpose of this study was aimed at reducing n-6:n-3 ratio of pork, which is better for consumer health, and improving meat quality by supplemented dietary $n-3$ PUFA rich oils accompany with vitamin $\mathrm{E}$ in pigs.

\section{Materials and Methods}

\subsection{Animal Treatment}

Sixty Landrace $\times$ Yorkshire $\times$ Duroc (LYD) finishing pigs (average body weight was $66.5 \pm 5.2 \mathrm{~kg}$ ) were allotted five dietary treatments with four replicates (pen), based on body weight and sex (half male and half female). They are (1) Lard (L), (2) Soybean oil (SO), (3) contain linseed commercial fish product, (CFP) (J. John, Co. Taiwan), (4) Canola oil (CO), (5) 50\% Fish oil $+50 \%$ Canola oil, (FCO). Dietary divided to growing (under $80 \mathrm{~kg}$ ) and finishing periods. Pigs were allowed to consume feed (Table 1) (NRC, 1998) and water ad libitum. The fatty acids composition of each group was listed in Tables 2 and 3, calculated dietary n-6:n-3 ratio at growing period are 14.46, 9.03, 6.52, 7.79 and 4.36, at finishing period are 16.63, 8.69, 7.21, 7.38 and 4.23 in group 1, 2, 3, 4 and 5, respectively. All the dietary are added $200 \mathrm{mg} / \mathrm{kg}$ of vitamin E. The experiment was conducted for 90 days. Blood samples were collected at the $60^{\text {th }}$ day, pigs were fast overnight before blood sampling; then at final of experiment 8 pigs of each group ( 4 male and 4 female) were selected for sacrificed and measured the carcass characteristics. Loin and belly muscle samples were used for meat traits analysis. Back fat and belly fat samples were used for fatty acids analysis. The experimental animals were reared following the guidelines in the Guide for the Care and Use of Agricultural Animals in Agricultural Research and Teaching. 
Table 1. Composition of basal diet

\begin{tabular}{|c|c|c|c|c|}
\hline & \multicolumn{2}{|c|}{$50-80 \mathrm{~kg}$} & \multicolumn{2}{|c|}{$80-120 \mathrm{~kg}$} \\
\hline & DO & CFP & DO & CFP \\
\hline \multicolumn{5}{|l|}{ Ingredients $(\mathrm{g} / \mathrm{kg})$} \\
\hline Corn & 684.7 & 661.7 & 746 & 713 \\
\hline Soybean meal & 175 & 175 & 110 & 110 \\
\hline Fish meal $(60 \% \mathrm{CP})$ & 25 & 0 & 15 & 0 \\
\hline Commercial fish product & 0 & 55 & 0 & 55 \\
\hline Wheat bran & 50 & 50 & 70 & 70 \\
\hline Calcium phosphate monobasic & 4.6 & 4.6 & 1 & 1 \\
\hline Limestone & 9.2 & 9.2 & 8.5 & 8.5 \\
\hline Oil & 46 & 39 & 44 & 37 \\
\hline Salt & 5 & 5 & 5 & 5 \\
\hline Vitamin premix $^{a}$ & 0.2 & 0.2 & 0.2 & 0.2 \\
\hline Mineral premix ${ }^{b}$ & 0.3 & 0.3 & 0.3 & 0.3 \\
\hline Total & 1000 & 1000 & 1000 & 1000 \\
\hline \multicolumn{5}{|l|}{ Calculated value } \\
\hline Metabolism energy (MJ/kg) & 13.40 & 13.40 & 13.46 & 13.48 \\
\hline Crude protein $(\%)$ & 16.11 & 16.17 & 13.46 & 13.69 \\
\hline \multicolumn{5}{|l|}{ Analyzed value } \\
\hline Crude fat $(\%)$ & 7.57 & 7.52 & 6.89 & 7.14 \\
\hline
\end{tabular}

Note. ${ }^{\text {a }}$ Vitamin premix supplied per kilogram contain: retinol 50,000,000 IU; cholecalciferol 6,250,000 IU; $\alpha$-tocopherol $160 \mathrm{~g}$; menadione $8 \mathrm{~g}$; thiamine $10 \mathrm{~g}$; riboflavin $20 \mathrm{~g}$; pyridoxine $16 \mathrm{~g}$; cyanocobalamin $0.16 \mathrm{~g}$; niacin $95 \mathrm{~g}$; pantothenic acid $60 \mathrm{~g}$; biotin $0.5 \mathrm{mg}$; folic acid: $7.5 \mathrm{~g}$.

${ }^{\mathrm{b}}$ Mineral premix supplied per kilogram contain: Fe 150 g; Cu 30 g; Mn 60 g; Zn: 120 g; Se 0.15 g; Co 0.7 g; I $1.5 \mathrm{~g}$.

$\mathrm{DO}=$ Different oil groups, $\mathrm{CFP}=$ Commercial fish product containing linseed group $(31.82 \% \mathrm{CP})$. 
Table 2. Fatty acid composition of 50-80 kg BW basal diet

\begin{tabular}{|c|c|c|c|c|c|}
\hline Fatty acids & Lard & $\mathrm{SO}$ & CFP & $\mathrm{CO}$ & FCO \\
\hline & \multicolumn{5}{|c|}{ 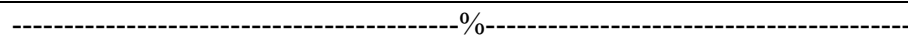 } \\
\hline C6:0 & 0.01 & 0.04 & 0.17 & 0.03 & 0.02 \\
\hline $\mathrm{C} 8: 0$ & 0.01 & 0.02 & 0.11 & 0.02 & 0.02 \\
\hline $\mathrm{C} 10: 0$ & 0.05 & - & 0.02 & 0.01 & 0.01 \\
\hline C12:0 & 0.07 & 0.01 & 0.03 & 0.04 & 0.06 \\
\hline C14:0 & 1.14 & 0.15 & 0.16 & 0.25 & 1.48 \\
\hline C15:0 & 0.11 & 0.03 & 0.04 & 0.04 & 0.12 \\
\hline C16:0 & 21.01 & 12.97 & 11.51 & 12.05 & 12.53 \\
\hline C17:0 & 0.34 & 0.12 & 0.09 & 0.11 & 0.18 \\
\hline C18:0 & 9.27 & 3.69 & 2.75 & 3.43 & 3.00 \\
\hline C20:0 & 0.28 & 0.38 & 0.68 & 0.42 & 0.44 \\
\hline $\mathrm{C} 21: 0$ & - & 0.06 & 0.06 & 0.06 & 0.03 \\
\hline $\mathrm{C} 22: 0$ & 0.09 & 0.30 & 0.40 & 0.31 & 0.22 \\
\hline $\mathrm{C} 23: 0$ & - & 0.04 & 0.06 & 0.04 & - \\
\hline $\mathrm{C} 24: 0$ & 0.07 & 0.17 & 0.23 & 0.18 & - \\
\hline SFA & 32.45 & 17.98 & 16.31 & 16.99 & 18.12 \\
\hline C14:1 & 0.06 & - & - & - & 0.02 \\
\hline C16:1 & 1.45 & 0.19 & 0.28 & 0.29 & 1.9 \\
\hline C18:1 & 33.67 & 23.63 & 53.54 & 29.39 & 38.38 \\
\hline C20:1 & 0.51 & 0.26 & 0.96 & 0.34 & 1.18 \\
\hline $\mathrm{C} 22: 1$ & 0.03 & - & - & - & 0.10 \\
\hline $\mathrm{C} 24: 1$ & - & - & 0.11 & 0.03 & 0.14 \\
\hline MUFA & 35.72 & 24.08 & 54.89 & 30.05 & 41.72 \\
\hline $\mathrm{C} 18: 2, \mathrm{n}-6$ & 29.51 & 51.92 & 25.54 & 46.58 & 32.23 \\
\hline $\mathrm{C} 18: 3, \mathrm{n}-3$ & 1.34 & 5.64 & 3.21 & 5.94 & 3.71 \\
\hline $\mathrm{C} 20: 2, \mathrm{n}-6$ & 0.31 & 0.04 & 0.05 & 0.04 & 0.26 \\
\hline $\mathrm{C} 20: 3, \mathrm{n}-6$ & 0.14 & - & - & - & 0.13 \\
\hline $\mathrm{C} 20: 4, \mathrm{n}-6$ & 0.12 & 0.01 & - & 0.02 & 0.15 \\
\hline $\mathrm{C} 20: 5, \mathrm{n}-3$ & 0.11 & 0.12 & - & 0.17 & 1.83 \\
\hline $\mathrm{C} 22: 2, \mathrm{n}-6$ & - & - & - & - & 0.02 \\
\hline $\mathrm{C} 22: 6, \mathrm{n}-3$ & 0.3 & 0.21 & - & 0.18 & 1.84 \\
\hline PUFA & 31.83 & 57.94 & 28.80 & 52.96 & 40.17 \\
\hline$n-6: n-3$ & 17.19 & 8.71 & 7.97 & 7.41 & 4.44 \\
\hline
\end{tabular}

Note. SFA: saturated fatty acids. MUFA: monounsaturated fatty acids.

$\mathrm{SO}=$ Soybean oil. $\mathrm{CFP}=$ Commercial fish product. $\mathrm{CO}=$ Canola oil. $\mathrm{FCO}=50 \%$ Fish oil $+50 \%$ Canola oil. 
Table 3. Fatty acid composition of $80-120 \mathrm{~kg}$ BW basal diet

\begin{tabular}{|c|c|c|c|c|c|}
\hline Fatty acids & Lard & $\mathrm{SO}$ & CFP & $\mathrm{CO}$ & FCO \\
\hline & \multicolumn{5}{|c|}{ 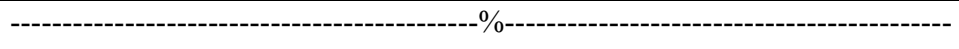 } \\
\hline C6:0 & 0.03 & 0.03 & 0.03 & 0.02 & 0.02 \\
\hline C8:0 & 0.01 & 0.01 & 0.02 & 0.01 & 0.01 \\
\hline C10:0 & - & - & 0.01 & 0.01 & 0.01 \\
\hline C12:0 & 0.01 & 0.01 & 0.05 & 0.06 & 0.05 \\
\hline $\mathrm{C} 14: 0$ & 0.16 & 0.16 & 0.12 & 0.25 & 1.49 \\
\hline C15:0 & 0.20 & 0.03 & 0.03 & 0.03 & 0.12 \\
\hline C16:0 & 20.35 & 12.73 & 12.43 & 11.79 & 12.06 \\
\hline C17:0 & 0.50 & 0.12 & 0.07 & 0.11 & 0.17 \\
\hline C18:0 & 8.90 & 3.71 & 2.06 & 3.27 & 2.75 \\
\hline C20:0 & 0.35 & 0.35 & 0.54 & 0.37 & 0.40 \\
\hline $\mathrm{C} 21: 0$ & 0.07 & 0.07 & 0.05 & 0.07 & 0.06 \\
\hline $\mathrm{C} 22: 0$ & 0.30 & 0.30 & 0.30 & 0.29 & 0.22 \\
\hline $\mathrm{C} 23: 0$ & - & 0.05 & 0.04 & 0.05 & - \\
\hline $\mathrm{C} 24: 0$ & 0.13 & 0.16 & 0.18 & 0.19 & - \\
\hline SFA & 32.05 & 17.73 & 15.89 & 16.52 & 17.37 \\
\hline C14:1 & 0.16 & - & - & - & 0.02 \\
\hline C16:1 & 1.51 & 0.21 & 0.26 & 0.27 & 1.95 \\
\hline C18:1 & 31.97 & 22.65 & 50.62 & 28.7 & 37.12 \\
\hline C20:1 & 0.4 & 0.26 & 0.92 & 0.30 & 1.21 \\
\hline $\mathrm{C} 22: 1$ & 0.04 & - & 0.05 & - & 0.12 \\
\hline $\mathrm{C} 24: 1$ & - & - & 0.13 & - & 0.14 \\
\hline MUFA & 34.08 & 23.12 & 51.98 & 29.27 & 40.56 \\
\hline $\mathrm{C} 18: 2, \mathrm{n}-6$ & 31.47 & 53.18 & 28.41 & 47.63 & 33.78 \\
\hline $\mathrm{C} 18: 3, \mathrm{n}-3$ & 1.81 & 5.56 & 3.3 & 6.16 & 3.82 \\
\hline $\mathrm{C} 20: 2, \mathrm{n}-6$ & 0.16 & 0.05 & 0.07 & 0.05 & 0.29 \\
\hline $\mathrm{C} 20: 3, \mathrm{n}-6$ & 0.06 & - & - & - & 0.14 \\
\hline $\mathrm{C} 20: 4, \mathrm{n}-6$ & 0.04 & 0.02 & 0.01 & 0.02 & 0.15 \\
\hline $\mathrm{C} 20: 5, \mathrm{n}-3$ & 0.11 & 0.12 & 0.11 & 0.18 & 1.92 \\
\hline $\mathrm{C} 22: 6, \mathrm{n}-3$ & 0.21 & 0.22 & 0.23 & 0.17 & 1.96 \\
\hline PUFA & 33.86 & 59.15 & 32.13 & 54.21 & 42.08 \\
\hline$n-6: n-3$ & 14.90 & 9.03 & 7.83 & 7.33 & 4.46 \\
\hline
\end{tabular}

\subsection{Growth Performance}

Pigs were weighed at the start and end of the experiment to calculate daily body weight gain. Feed consumption was recorded for calculating feed intake, and the feed conversion ratio.

\subsection{Blood Traits}

Serum cholesterol and triglyceride (TG) were determined by commercial enzymatic test kit (Roche/Hitachi) and determined photometrically using a serum biochemical auto-analyzer (Roche, Co. Switzerland).

Lipoprotein profile (including HDL, LDL and VLDL) was determined by electrophoresis method using TITAN GEL Lipoprotein Kit (3045, Helena Laboratories, Texas). After drying, the gels were stained, then destained, and then scanned with a densitometer at $525 \mathrm{~nm}$ (Helena Co. 8JF00105, US). Non-esterified fatty acid (NEFA) concentrations were determined using a commercial enzymatic kit procedure (Biovision, USA).

High-density lipoprotein cholesterol (HDL-C) determination was based on the method described by Dobiasova et al. (1991). Low-density lipoprotein cholesterol (LDL-C) was determined according to Dobiasova et al. (1991) method. 


\subsection{Carcass Characteristic}

Carcass yield was calculated based on carcass measurements of carcass weight over live weight. Average back fat thickness (skinless) was measured at the shoulder, last costal and lumbar rib in the unit $\mathrm{cm} / 100 \mathrm{~kg}$. In the longissimus muscle area, the carcass was straight cut between the tenth and eleventh rib of the longissimus muscle, and the area drawn on tracing paper, then the integral was used to calculate the area in the unit $\mathrm{mm}^{2} / 100$ $\mathrm{kg}$.

\subsection{Muscle Fat Content}

Muscle fat content was determined follow the Folch et al. (1957) method. Muscle samples of $20 \mathrm{~g}$ were immersed in $200 \mathrm{~mL}$ solvent (chloroform:methanol = 2:1) overnight, and then filtered. Next, $40 \mathrm{~mL} 0.9 \% \mathrm{NaCl}$ solution was added into a separating funnel. The lower layer of liquid was fat and chloroform. After drying, the samples were weighed and the fat content was calculated.

\subsection{Color Difference}

Meat Hunter L, a, b values were measured duplicate using a color difference meter (Model TC-1, Tokyo Denshoku Co., LTD.). L, a and b values represent light, amaranth and beige, respectively.

\subsection{Water Holding Capacity}

Water holding capacity (WHC) was determined duplicate using the Lesiak et al. (1997) method. $10 \mathrm{~mL}$ of a $1 \%$-sodium chloride solution was added to $5 \mathrm{~g}$ samples of breast and thigh muscle, placed in a water bath at $70-75{ }^{\circ} \mathrm{C}$ for $30 \mathrm{~min}$, cooled and centrifuged at $12235 \times \mathrm{g}$ (Himac SCR 20B, Hitachi, Japan). The supernatant was then collected and weighed. Water holding capacity was then calculated.

\subsection{Fatty Acids Analysis}

The samples esterification was added $5 \mathrm{ml} 0.5 \mathrm{~N}$ methanolic-NaOH and boiling for 5 mins with a cooling equipment, then added $5 \mathrm{~mL} \mathrm{BF}_{3}-\mathrm{MeOH}$ and boiled for 2 mins. Next, $5 \mathrm{~mL}$ heptane was added and boiled for 1 min. After cool then added saturated $\mathrm{NaCl}$ to let the esterified fatty acid-heptane layer separated and taken for fatty acids analysis (Prabhakara Rao et al., 2010).

Fatty acids analysis was carried out with a FID detector gas chromatography (Agilent 6890N, USA) with DB-23 column. The initial temperature was $60^{\circ} \mathrm{C}$ and maintain for 2 mins then elevate to $210{ }^{\circ} \mathrm{C}$ maintain for 7 mins. FID detector temperature was $280^{\circ} \mathrm{C}$, carrier air was nitrogen, flow rate was $1.5 \mathrm{~mL} / \mathrm{min}$.

\subsection{Sensory Evaluation}

Samples of breast and thigh muscle, respectively, were cooked at $80{ }^{\circ} \mathrm{C}$ for $15 \mathrm{~min}$, and a piece of $1 \mathrm{~cm}$ was cut off for evaluation of appearance, flavor, tenderness, juiciness and overall acceptability by 50 persons. Scoring was between 1 and 7 , with 7 representing the best grade.

\subsection{Meat Storage Test}

Meat samples were stored at $-20^{\circ} \mathrm{C}$ for 15 days, then for antioxidation traits determine.

Thiobarbituric acid-reactive substances (TBARS) were determined based on the procedure reported by Fraga et al. (1988). The scavenging DPPH (a,a-diphenyl- $\beta$-picrylhydrazyl) radical ability was determined in triplicate, following the method reported by Chung et al. (2002). Peroxide value (POV) was determined following the procedure described by Sebranek (1978). Conjugated diene hydroperoxide contents were measured according the method described by Osawa et al. (1992).

\subsection{Statistical Analysis}

The experimental data was subjected to analysis of variance using general linear model procedures (PROC GLM) of SAS (1998). Tukey's test was used to determine significant differences between treatment means. According to the following model, $Y=\mu+T_{i}+P_{j}+e_{i j k}$, treatment (T) was the main effect. Where $Y$ is the dependent variable, $\mu$ represents the mean, $\mathrm{P}$ is the pen (replicate) effect and $\mathrm{e}$ is the random residual error term. 


\section{Results and Discussion}

\subsection{Growth Performances of Finishing-Pigs in Response to Dietary Supplementation of Different Oils (Table 4)}

Table 4. Growth performances of pigs in response to dietary supplementation of different oil

\begin{tabular}{lllllll}
\hline Items & Lard & SO & CFP & CO & FCO & SEM \\
\hline Initial body weight $(\mathrm{kg})$ & 63.22 & 65.91 & 63.50 & 63.69 & 64.11 & 1.83 \\
Final body weight (kg) & 118.4 & 117.6 & 124.0 & 120.0 & 116.9 & 2.70 \\
Average daily gain (kg/day) & 0.61 & 0.59 & 0.65 & 0.63 & 0.59 & 0.04 \\
Average feed intake (kg/day) & 2.26 & 2.09 & 2.18 & 2.12 & 2.15 & 0.08 \\
Feed efficiency (feed/gain) & 3.68 & 3.64 & 3.25 & 3.39 & 3.67 & 0.16
\end{tabular}

Note. $\mathrm{n}=4 . \mathrm{SO}=$ Soybean oil; $\mathrm{CFP}=$ Commercial fish product; $\mathrm{CO}=$ Canola oil; $\mathrm{FCO}=50 \%$ Fish oil $+50 \%$ Canola oil.

The results showed that different dietary oils had no influence on final body weight, average feed intake and feed efficiency $(\mathrm{P}>0.05)$ of finishing-pigs. Previous reports indicated that under similar nutritional concentrations, different oils supplementation did not affect average daily gain, average feed intake and feed efficiency of animals (Thacker, 1998; Teye et al., 2006; Mitchaothai et al., 2007).

3.2 Carcass Characteristics of Finishing-Pigs in Response to Dietary Supplementation of Different Oil (Table 5)

Table 5. Carcass characteristics of pigs in response to dietary supplementation of different oil

\begin{tabular}{lllllll}
\hline Items & Lard & SO & CFP & CO & FCO & SEM \\
\hline Carcass weight $(\mathrm{kg})$ & 99.81 & 98.89 & 102.9 & 100.9 & 100.6 & 3.87 \\
Carcass yield (\%) & 84.8 & 86.1 & 85.9 & 86.0 & 86.3 & 0.50 \\
Back fat thickness (cm) & 2.14 & 2.01 & 2.17 & 1.92 & 2.10 & 0.11 \\
Lion-eye area $\left(\mathrm{cm}^{2}\right)$ & 52.70 & 52.84 & 52.09 & 51.73 & 49.56 & 2.97 \\
$\mathrm{pH}_{1}$ & 6.51 & 6.28 & 6.36 & 6.30 & 6.50 & 0.10 \\
$\mathrm{pH}_{24}$ & 5.73 & 5.83 & 5.89 & 5.78 & 5.81 & 0.09
\end{tabular}

Note. $\mathrm{n}=4 . \mathrm{SO}=$ Soybean oil; $\mathrm{CFP}=$ Commercial fish product; $\mathrm{CO}=$ Canola oil; $\mathrm{FCO}=50 \%$ Fish oil $+50 \%$ Canola oil.

Different dietary oils had no influence on carcass weight, carcass yield, back fat thickness, meat $\mathrm{pH}$ value at $1 \mathrm{~h}$ $\left(\mathrm{pH}_{1}\right)$ and $24 \mathrm{~h}\left(\mathrm{pH}_{24}\right)$ after been slaughtered of finishing-pigs (Table 5; $\left.\mathrm{P}>0.05\right)$. The results agreed with the observations in growing-pigs (Thacker, 1998; Teye et al., 2006), and finishing-pig (Mitchaothai et al., 2007). All these results indicated that different dietary oils supplementation would not influence the carcass quality and characteristics of finishing-pigs. 
3.3 Plasma Parameters of Finishing-Pigs in Response to Dietary Supplementation of Different Oil (Table 6)

Table 6. Plasma parameters of pigs in response to dietary supplementation of different oil

\begin{tabular}{|c|c|c|c|c|c|c|}
\hline Items & Lard & $\mathrm{SO}$ & CFP & $\mathrm{CO}$ & $\mathrm{FCO}$ & SEM \\
\hline Total cholesterol $(\mathrm{mg} / \mathrm{dL})$ & $138.7^{\mathrm{a}}$ & $125.1^{\mathrm{ab}}$ & $125.8^{\mathrm{ab}}$ & $132.7^{\mathrm{ab}}$ & $116.65^{\mathrm{b}}$ & 5.71 \\
\hline HDL-C (mg/dL) & $8.30^{\mathrm{ab}}$ & $6.80^{\mathrm{b}}$ & $8.09^{\mathrm{ab}}$ & $8.77^{\mathrm{a}}$ & $9.25^{\mathrm{a}}$ & 0.48 \\
\hline LDL-C (mg/dL) & $109.63^{\mathrm{a}}$ & $97.89^{\mathrm{ab}}$ & $99.04^{\mathrm{ab}}$ & $105.4^{\mathrm{ab}}$ & $89.27^{\mathrm{b}}$ & 5.03 \\
\hline Triglyceride (mg/dL) & $621.5^{\mathrm{b}}$ & $1000.0^{\mathrm{a}}$ & $1003.9^{\mathrm{a}}$ & $708.2^{\mathrm{ab}}$ & $651.0^{\mathrm{b}}$ & 99.1 \\
\hline $\mathrm{NEFA}(\mu \mathrm{mol} / \mathrm{L})$ & $771.1^{\mathrm{ab}}$ & $639.1^{\mathrm{b}}$ & $600.6^{\mathrm{b}}$ & $824.7^{\mathrm{a}}$ & $738.9^{\mathrm{ab}}$ & 53.7 \\
\hline \multicolumn{7}{|l|}{ Lipoprotein Profile } \\
\hline HDL (\%) & $49.06^{\mathrm{b}}$ & $46.64^{\mathrm{b}}$ & $47.32^{\mathrm{b}}$ & $49.08^{\mathrm{b}}$ & $55.84^{\mathrm{a}}$ & 1.16 \\
\hline VLDL (\%) & $8.75^{\mathrm{c}}$ & $14.95^{\mathrm{a}}$ & $14.62^{\mathrm{ab}}$ & $12.38^{\mathrm{abc}}$ & $10.97^{b c}$ & 1.17 \\
\hline LDL (\%) & $42.13^{\mathrm{a}}$ & $38.40^{\mathrm{b}}$ & $38.04^{\mathrm{b}}$ & $38.55^{\mathrm{b}}$ & $33.26^{\mathrm{c}}$ & 0.95 \\
\hline
\end{tabular}

Note. ${ }^{\mathrm{a}, \mathrm{b}, \mathrm{c}, \mathrm{d}}$ Means within the same row without a common superscript differ significantly $(\mathrm{P}<0.05) . \mathrm{n}=4$. HDL-C: high-density lipoprotein-Cholesterol; LDL-C: low-density lipoprotein-Cholesterol; NEFA: nonesterified fatty acids; $\mathrm{SO}=$ Soybean oil; $\mathrm{CFP}=$ Commercial fish product; $\mathrm{CO}=$ Canola oil; $\mathrm{FCO}=50 \%$ Fish oil $+50 \%$ Canola oil.

Cholesterol content in FCO group was lower than that in lard group (Table 6; $\mathrm{P}<0.05$ ), lard group was tend to have higher cholesterol than SO and CGP groups, the results suggested that lard could increase the cholesterol in plasma while fish oil decreased. The data obtained in this trial did not agree with the Byoung et al. (1997) and Lien et al. (2003) reports, who indicated that there existed no difference in plasma cholesterol among different dietary oils supplementation.

Plasma HDL-C in CO and FCO groups was higher than in SO group (Table 6; $\mathrm{P}<0.05$ ), plasma LDL-C in FCO was lower than Lard groups $(\mathrm{P}<0.05)$, the results were similar to the observations of Lien et al. (2003) and Riediger et al. (2008), who supplied fish oil into diets of hens and mice respectively. Chang et al. (2010) also reported that saturated fat (SAT) diets increased, but n-3 diets decreased the arterial low-density lipoprotein (LDL) cholesterol deposition.

Plasma TG in lard and FCO group were significantly lower than SO and CFP groups $(\mathrm{P}<0.05)$. Concerning about the lipoprotein profile, FCO group had highest HDL and lowest LDL ratio than other groups $(\mathrm{P}<0.05)$, which was similar to the report of Lien et al. (2003). Lard group had highest plasma LDL than other groups as well as cholesterol content $(\mathrm{P}<0.05)$. The SO and CFP groups had lower NEFA concentrations than that in $\mathrm{CO}$ group $(\mathrm{P}<0.05)$, and tend to be lower than FCO group.

From the plasma parameter indicated that the FCO group, the lower n-6:n-3 ratio PUFA are reduced plasma cholesterol, LDL and LDL-C, those bad factors for artery and heart disease; meanwhile enhanced HDL and HDL-C, the benefit factors for artery and heart disease. Thus, lower n-6:n-3 ratio PUFA is good for consumer health. 
3.4 Fatty Acid Compositions of Back Fat and Belly Meat of Finishing-Pigs in Response to Dietary Supplementation of Different Oil (Tables 7 and 8)

Table 7. Back fat fatty acid composition of pigs in response to dietary supplementation of different oil

\begin{tabular}{|c|c|c|c|c|c|c|}
\hline Fatty acids & Lard & SO & CFP & $\mathrm{CO}$ & FCO & SEM \\
\hline & \multicolumn{6}{|c|}{ 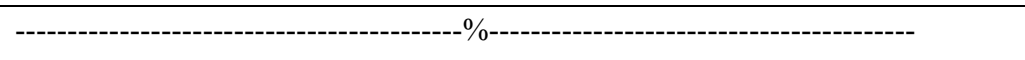 } \\
\hline C6:0 & 0.16 & 0.18 & 0.07 & 0.10 & 0.12 & 0.04 \\
\hline C8:0 & $0.14^{\mathrm{a}}$ & $0.12^{\mathrm{ab}}$ & $0.05^{\mathrm{b}}$ & $0.06^{\mathrm{b}}$ & $0.10^{\mathrm{ab}}$ & 0.03 \\
\hline $\mathrm{C} 10: 0$ & 0.11 & 0.11 & 0.10 & 0.10 & 0.12 & 0.01 \\
\hline $\mathrm{C} 12: 0$ & 0.14 & 0.12 & 0.13 & 0.12 & 0.13 & 0.01 \\
\hline C14:0 & $1.72^{\mathrm{a}}$ & $1.55^{\mathrm{ab}}$ & $1.47^{\mathrm{b}}$ & $1.41^{\mathrm{b}}$ & $1.71^{\mathrm{a}}$ & 0.06 \\
\hline $\mathrm{C} 15: 0$ & $0.09^{\mathrm{a}}$ & $0.09^{\mathrm{ab}}$ & $0.06^{\mathrm{b}}$ & $0.08^{\mathrm{ab}}$ & $0.09^{\mathrm{ab}}$ & 0.01 \\
\hline C16:0 & $27.80^{\mathrm{a}}$ & $25.83^{\mathrm{a}}$ & $23.35^{\mathrm{b}}$ & $22.06^{\mathrm{b}}$ & $23.34^{\mathrm{a}}$ & 0.77 \\
\hline C17:0 & $0.45^{\mathrm{a}}$ & $0.40^{\mathrm{ab}}$ & $0.30^{\mathrm{b}}$ & $0.35^{\mathrm{ab}}$ & $0.38^{\mathrm{ab}}$ & 0.04 \\
\hline C18:0 & $13.99^{\mathrm{a}}$ & $12.8^{\mathrm{a}}$ & $10.79^{b}$ & $10.61^{b}$ & $10.69^{\mathrm{b}}$ & 0.47 \\
\hline C20:0 & $0.26^{\mathrm{ab}}$ & $0.26^{\mathrm{ab}}$ & $0.23^{\mathrm{b}}$ & $0.25^{\mathrm{ab}}$ & $0.27^{\mathrm{a}}$ & 0.01 \\
\hline SFA & $44.86^{\mathrm{a}}$ & $41.45^{\mathrm{a}}$ & $35.56^{\mathrm{b}}$ & $35.14^{\mathrm{b}}$ & $36.95^{b}$ & 1.31 \\
\hline C14:1 & $0.019^{\mathrm{a}}$ & $0.015^{\mathrm{b}}$ & $0.015^{\mathrm{b}}$ & $0.014^{\mathrm{b}}$ & $0.014^{b}$ & 0.001 \\
\hline C16:1 & $1.71^{\mathrm{b}}$ & $1.97^{\mathrm{ab}}$ & $1.87^{\mathrm{ab}}$ & $2.04^{\mathrm{a}}$ & $1.70^{\mathrm{b}}$ & 0.10 \\
\hline C18:1 & $40.49^{\mathrm{a}}$ & $36.85^{\mathrm{b}}$ & $37.95^{\mathrm{b}}$ & $43.59^{\mathrm{a}}$ & $38.59^{\mathrm{ab}}$ & 0.90 \\
\hline C20:1 & $0.81^{\mathrm{b}}$ & $0.71^{\mathrm{c}}$ & $0.66^{\mathrm{c}}$ & $0.88^{\mathrm{a}}$ & $0.90^{\mathrm{a}}$ & 0.02 \\
\hline MUFA & $43.29^{\mathrm{a}}$ & $39.29^{b}$ & $40.50^{\mathrm{b}}$ & $46.54^{\mathrm{a}}$ & $43.20^{\mathrm{a}}$ & 0.96 \\
\hline $\mathrm{C} 18: 2, \mathrm{n}-6$ & $10.65^{\mathrm{c}}$ & $17.20^{\mathrm{ab}}$ & $20.83^{\mathrm{a}}$ & $15.01^{\mathrm{bc}}$ & $16.82^{\mathrm{ab}}$ & 1.54 \\
\hline $\mathrm{C} 18: 3, \mathrm{n}-3$ & $0.49^{c}$ & $1.10^{\mathrm{bc}}$ & $1.83^{\mathrm{a}}$ & $2.04^{\mathrm{ab}}$ & $1.94^{\mathrm{a}}$ & 0.22 \\
\hline $\mathrm{C} 20: 2, \mathrm{n}-6$ & $0.43^{\mathrm{b}}$ & $0.62^{\mathrm{ab}}$ & $0.73^{\mathrm{a}}$ & $0.61^{\mathrm{ab}}$ & $0.43^{\mathrm{b}}$ & 0.06 \\
\hline$C 20: 3, n-6^{*}$ & 0.048 & 0.048 & 0.075 & 0.054 & 0.068 & 0.01 \\
\hline $\mathrm{C} 20: 3, \mathrm{n}-3^{* *}$ & $0.09^{\mathrm{b}}$ & $0.14^{\mathrm{ab}}$ & $0.21^{\mathrm{a}}$ & $0.20^{\mathrm{a}}$ & $0.10^{\mathrm{b}}$ & 0.03 \\
\hline $\mathrm{C} 20: 4, \mathrm{n}-6$ & 0.10 & 0.10 & 0.15 & 0.15 & 0.09 & 0.03 \\
\hline $\mathrm{C} 20: 5, \mathrm{n}-3$ & $0.020^{\mathrm{c}}$ & $0.018^{\mathrm{c}}$ & $0.034^{\mathrm{bc}}$ & $0.136^{\mathrm{ab}}$ & $0.184^{\mathrm{a}}$ & 0.03 \\
\hline $\mathrm{C} 22: 6, \mathrm{n}-3$ & $0.05^{\mathrm{b}}$ & $0.06^{\mathrm{b}}$ & $0.07^{\mathrm{b}}$ & $0.12^{\mathrm{ab}}$ & $0.22^{\mathrm{a}}$ & 0.05 \\
\hline PUFA & $11.88^{\mathrm{c}}$ & $19.29^{\mathrm{ab}}$ & $23.94^{\mathrm{a}}$ & $18.32^{\mathrm{b}}$ & $19.85^{\mathrm{ab}}$ & 1.89 \\
\hline$n-6: n-3$ & $17.18^{\mathrm{a}}$ & $13.63^{\mathrm{ab}}$ & $10.16^{\mathrm{b}}$ & $6.34^{\mathrm{c}}$ & $7.12^{\mathrm{c}}$ & 1.63 \\
\hline
\end{tabular}

Note. ${ }^{\mathrm{a}, \mathrm{b}, \mathrm{c}}$ Means within the same row without a common superscript differ significantly $(\mathrm{P}<0.05) . \mathrm{n}=4$.

* cis-8,11,14-Eicosatrienoic acid. ** cis-11,14,17-Eicosatrienoic acid.

$\mathrm{SO}=$ Soybean oil. $\mathrm{CFP}=$ Commercial fish product. $\mathrm{CO}=$ Canola oil. $\mathrm{FCO}=50 \%$ Fish oil $+50 \%$ Canola oil. 
Table 8 . Belly meat fatty acid composition of pigs in response to dietary supplementation of different oil

\begin{tabular}{|c|c|c|c|c|c|c|}
\hline Fatty acids & Lard & SO & CFP & $\mathrm{CO}$ & FO & SEM \\
\hline & \multicolumn{6}{|c|}{ |-----------------------------------------------\%o----------------------------------------- } \\
\hline $\mathrm{C} 10: 0$ & 0.11 & 0.10 & 0.11 & 0.12 & 0.12 & 0.01 \\
\hline $\mathrm{C} 12: 0$ & 0.12 & 0.10 & 0.12 & 0.11 & 0.11 & 0.01 \\
\hline C14:0 & $1.51^{\mathrm{a}}$ & $1.31^{\mathrm{b}}$ & $1.45^{\mathrm{ab}}$ & $1.43^{\mathrm{ab}}$ & $1.54^{\mathrm{a}}$ & 0.06 \\
\hline $\mathrm{C} 15: 0$ & $0.056^{\mathrm{ab}}$ & $0.063^{\mathrm{a}}$ & $0.046^{\mathrm{b}}$ & $0.055^{\mathrm{ab}}$ & $0.059^{\mathrm{ab}}$ & 0.04 \\
\hline $\mathrm{C} 16: 0$ & 24.46 & 22.36 & 23.46 & 22.62 & 23.50 & 0.96 \\
\hline $\mathrm{C} 17: 0$ & $0.30^{\mathrm{a}}$ & $0.32^{\mathrm{a}}$ & $0.24^{\mathrm{b}}$ & $0.27^{\mathrm{ab}}$ & $0.27^{\mathrm{ab}}$ & 0.02 \\
\hline C18:0 & $12.59^{\mathrm{a}}$ & $12.40^{\mathrm{ab}}$ & $11.92^{\mathrm{b}}$ & $11.31^{\mathrm{b}}$ & $12.29^{\mathrm{ab}}$ & 0.34 \\
\hline C20:0 & 0.21 & 0.21 & 0.22 & 0.21 & 0.22 & 0.01 \\
\hline SFA & $39.42^{\mathrm{a}}$ & $36.88^{\mathrm{ab}}$ & $37.27^{\mathrm{ab}}$ & $36.14^{\mathrm{b}}$ & $38.16^{\mathrm{ab}}$ & 1.07 \\
\hline C14:1 & $0.019^{\mathrm{a}}$ & $0.013^{\mathrm{b}}$ & $0.015^{\mathrm{ab}}$ & $0.016^{\mathrm{ab}}$ & $0.014^{\mathrm{ab}}$ & 0.002 \\
\hline C16:1 & $2.06^{\mathrm{a}}$ & $1.51^{\mathrm{b}}$ & $1.90^{\mathrm{a}}$ & $2.07^{\mathrm{a}}$ & $2.13^{\mathrm{a}}$ & 0.13 \\
\hline C18:1 & $39.71^{\mathrm{a}}$ & $34.56^{\mathrm{b}}$ & $35.58^{\mathrm{b}}$ & $39.92^{\mathrm{a}}$ & $39.72^{\mathrm{a}}$ & 0.70 \\
\hline $\mathrm{C} 20: 1$ & $0.77^{\mathrm{a}}$ & $0.67^{\mathrm{b}}$ & $0.63^{\mathrm{b}}$ & $0.80^{\mathrm{a}}$ & $0.81^{\mathrm{a}}$ & 0.02 \\
\hline MUFA & $42.66^{\mathrm{a}}$ & $36.78^{b}$ & $38.14^{\mathrm{b}}$ & $42.82^{\mathrm{a}}$ & $42.69^{\mathrm{a}}$ & 0.70 \\
\hline $\mathrm{C} 18: 2, \mathrm{n}-6$ & $15.60^{\mathrm{b}}$ & $22.74^{\mathrm{a}}$ & $20.81^{\mathrm{a}}$ & $17.56^{\mathrm{b}}$ & $15.57^{b}$ & 0.97 \\
\hline C18:3, n-3 & $1.12^{\mathrm{c}}$ & $2.00^{\mathrm{ab}}$ & $2.15^{\mathrm{a}}$ & $1.91^{\mathrm{ab}}$ & $1.68^{\mathrm{b}}$ & 0.14 \\
\hline$C 20: 2, n-6$ & $0.66^{\mathrm{bc}}$ & $0.82^{\mathrm{a}}$ & $0.74^{\mathrm{ab}}$ & $0.66^{\mathrm{bc}}$ & $0.60^{\mathrm{c}}$ & 0.03 \\
\hline C20:3, n-6* & 0.10 & 0.11 & 0.10 & 0.10 & 0.11 & 0.01 \\
\hline $\mathrm{C} 20: 3, \mathrm{n}-3^{* *}$ & $0.16^{\mathrm{c}}$ & $0.22^{\mathrm{ab}}$ & $0.24^{\mathrm{a}}$ & $0.23^{\mathrm{ab}}$ & $0.19^{\mathrm{bc}}$ & 0.01 \\
\hline $\mathrm{C} 20: 4, \mathrm{n}-6$ & 0.27 & 0.24 & 0.29 & 0.29 & 0.24 & 0.03 \\
\hline $\mathrm{C} 20: 5, \mathrm{n}-3$ & $0.09^{\mathrm{b}}$ & $0.04^{\mathrm{b}}$ & $0.05^{\mathrm{b}}$ & $0.08^{\mathrm{b}}$ & $0.28^{\mathrm{a}}$ & 0.03 \\
\hline$C 22: 6, n-3$ & $0.15^{\mathrm{b}}$ & $0.14^{\mathrm{b}}$ & $0.11^{\mathrm{b}}$ & $0.21^{\mathrm{b}}$ & $0.48^{\mathrm{a}}$ & 0.04 \\
\hline PUFA & $18.15^{\mathrm{c}}$ & $26.31^{\mathrm{a}}$ & $24.49^{\mathrm{ab}}$ & $21.04^{b c}$ & $19.15^{\mathrm{c}}$ & 1.17 \\
\hline$n-6: n-3$ & $10.94^{\mathrm{a}}$ & $9.96^{\mathrm{a}}$ & $8.60^{\mathrm{b}}$ & $7.66^{\mathrm{b}}$ & $6.28^{\mathrm{c}}$ & 0.46 \\
\hline
\end{tabular}

Note. ${ }^{\mathrm{a}, \mathrm{b}, \mathrm{c}}$ Means within the same row without a common superscript differ significantly $(\mathrm{P}<0.05) . \mathrm{n}=4$.

* cis-8,11,14-Eicosatrienoic acid. ** cis-11,14,17-Eicosatrienoic acid.

$\mathrm{SO}=$ Soybean oil. $\mathrm{CFP}=$ Commercial fish product. $\mathrm{CO}=$ Canola oil. $\mathrm{FCO}=50 \%$ Fish oil $+50 \%$ Canola oil.

In back fat, lard group obtained largest saturated fatty acid percentage, but was significantly higher $\mathrm{CO}$ group only (Table 7; $\mathrm{P}<0.05$ ). Lard, $\mathrm{CO}$ and FCO groups had higher monounsaturated fatty acids (MUFA) percentages than SO and CFP $(\mathrm{P}<0.05)$. Among all types of PUFA, linoleic acid $(\mathrm{C} 18: 2)$ was the most abundant in $n-6$ fatty acid, while linolenic acid (C18:3) was the most abundant in $n-3$ fatty acid. Although the CFP group had highest contain of linolenic acid, but it also had relatively higher linoleic acid content to make a high n-6:n-3 ratio (10.16). CO group contained significantly $(\mathrm{P}<0.05)$ lower linoleic acid than CFP group, and had lower n-6:n-3 ratio (6.34) than CFP group (10.16). However, which still could not meet the suggestion of Department of Health (UK), the n-6:n-3 ratio should be 4 .

Total PUFA contains in back fat of SO, CFP, CO and FCO group were higher than those of lard group $(\mathrm{P}<0.05)$. The n-6:n-3 ratio were also lower in CFP, CO and FCO groups than in lard group $(\mathrm{P}<0.05)$. Scientists are suggested that high PUFA content was not certainly advantageous for human health, it is depend on the n- $6: n-3$ ratio.

In belly meat, total SFA contain in lard group was higher than that in CO group $(\mathrm{P}<0.05)$, MUFA level in lard, $\mathrm{CO}$ and FCO groups were higher than those of SO and CFP groups $(\mathrm{P}<0.05)$. SO group had higher PUFA than lard, $\mathrm{CO}$ and FCO groups $(\mathrm{P}<0.05)$, The $\mathrm{n}-6: \mathrm{n}-3$ ratio were lower in CFP, $\mathrm{CO}$ and FCO groups than lard and SO groups $(\mathrm{P}<0.05)$.

SO and CFP group had higher linoleic acid among all treatments $(\mathrm{P}<0.05)$, lard group showed lowest linolenic acid level compared with each of the other four groups $(\mathrm{P}<0.05)$. FCO group obtain highest EPA $(\mathrm{C} 20: 5, \mathrm{n}-3)$ 
and DHA $(\mathrm{C} 22: 6, \mathrm{n}-3)$ than other groups $(\mathrm{P}<0.05)$, which resulted in the lowest $\mathrm{n}-6$ :n-3 ratio of $\mathrm{FCO}(\mathrm{P}<0.05)$. These results were similar to the experiment of García-Rebollar et al. (2008), who supplied fish oil into pullet diet. The n-6:n-3 ratio of CEP, and CO group were also lower than lard and SO group.

The fatty acids composition is sensitive the dietary fat. West and Myer (1987) reported feeding animal with rich of C18:2 (n-6 FA) soybean oil or sunflower oil, the animal body C18:2 from 10-15\% increased to $30 \%$. In order to reduce n-6:n-3 ratio have some reports using canola oil and linseed (Wood et al., 2003), and have the good effective, however, can not using high level (no more than $3 \%$ ), otherwise will have bad effect on meat flavor, since high amount of C18:3 will result of bad odours (Shackelford et al., 1990). Warnants et al. (1999) reported using the dietary fat to modify animal body fat is quickly, the feeding time about 40 days could have the largest effective. This study also have the effective, but the feeding time is 90 days still not reach the expect n-6:n-3 value of 4 . However, the effect would be more obvious as supplemental time more long.

3.5 Meat Quality and Sensory Evaluation of Finishing-Pigs in Response to Dietary Supplementation of Different Oil (Table 9, 10)

Table 9. Meat quality of pigs in response to dietary supplementation of different oil

\begin{tabular}{llllllll}
\hline Items & Lard & SO & CFP & CO & FCO & SEM \\
\hline L & 41.20 & 42.42 & 40.44 & 41.05 & 42.24 & 1.32 \\
$\mathrm{a}$ & 9.93 & 9.27 & 10.29 & 9.44 & 9.52 & 0.53 \\
$\mathrm{~b}$ & 7.51 & 7.55 & 7.43 & 7.34 & 7.33 & 0.35 \\
TBARS, MDA $(\mu \mathrm{g} / \mathrm{g})$ & $0.27^{\mathrm{ab}}$ & $0.32^{\mathrm{a}}$ & $0.31^{\mathrm{ab}}$ & $0.24^{\mathrm{b}}$ & $0.29^{\mathrm{ab}}$ & 0.02 \\
DPPH $(\%)$ & 49.78 & 49.95 & 49.41 & 50.39 & 49.15 & 0.60 \\
POV $(\%)$ & 99.76 & 100.1 & 99.21 & 99.21 & 98.69 & 0.76 \\
Water holding capacity $(\%)$ & 87.87 & 87.08 & 84.61 & 85.66 & 87.27 & 0.33 \\
Fat content $(\%)$ & 2.36 & 2.34 & 2.39 & 2.23 & 2.98 & 0.36 \\
\hline
\end{tabular}

Note. ${ }^{\mathrm{a}, \mathrm{b}}$ Means within the same row without a common superscript differ significantly $(\mathrm{P}<0.05) . \mathrm{n}=4$.

L: light; a: amaranth; b: beige; DPPH: a,a-diphenyl- $\beta$-picrylhydrazyl. POV: peroxide value.

$\mathrm{SO}=$ Soybean oil $\mathrm{CFP}=$ Commercial fish product. $\mathrm{CO}=$ Canola oil $. \mathrm{FCO}=50 \%$ Fish oil $+50 \%$ Canola oil.

Table 10. Sensory evaluation of meat from pigs in response to dietary supplementation of different oil

\begin{tabular}{|c|c|c|c|c|c|c|}
\hline Items & Lard & SO & CFP & $\mathrm{CO}$ & $\mathrm{FCO}$ & SEM \\
\hline \multicolumn{7}{|l|}{ Longissimus muscle } \\
\hline Color & 4.44 & 4.36 & 4.04 & 4.28 & 4.32 & 0.20 \\
\hline Smell & 3.96 & 3.92 & 3.96 & 4.00 & 3.80 & 0.24 \\
\hline Flavor & $3.80^{\mathrm{ab}}$ & $4.28^{\mathrm{a}}$ & $4.04^{\mathrm{ab}}$ & $3.40^{\mathrm{b}}$ & $4.04^{\mathrm{ab}}$ & 0.22 \\
\hline Texture & $3.48^{\mathrm{ab}}$ & $4.04^{\mathrm{a}}$ & $4.12^{\mathrm{a}}$ & $3.08^{\mathrm{b}}$ & $3.80^{\mathrm{a}}$ & 0.23 \\
\hline Juiciness & $3.36^{\mathrm{ab}}$ & $4.04^{\mathrm{a}}$ & $3.72^{\mathrm{a}}$ & $2.72^{\mathrm{b}}$ & $3.40^{\mathrm{ab}}$ & 0.26 \\
\hline Total acceptance & $3.67^{\mathrm{bc}}$ & $4.46^{\mathrm{a}}$ & $4.08^{\mathrm{ab}}$ & $3.08^{\mathrm{c}}$ & $3.92^{\mathrm{ab}}$ & 0.23 \\
\hline \multicolumn{7}{|l|}{ Belly meat } \\
\hline Color & 4.64 & 4.60 & 4.60 & 4.60 & 4.64 & 0.21 \\
\hline Smell & 4.16 & 4.56 & 4.40 & 4.36 & 4.24 & 0.22 \\
\hline Flavor & 4.40 & 4.72 & 4.68 & 4.28 & 4.44 & 0.22 \\
\hline Texture & 4.80 & 4.84 & 4.68 & 4.80 & 4.84 & 0.20 \\
\hline Juiciness & 4.96 & 5.00 & 4.92 & 4.92 & 5.00 & 0.22 \\
\hline Total acceptance & 4.58 & 4.83 & 5.00 & 4.79 & 4.50 & 0.24 \\
\hline
\end{tabular}

Note. ${ }^{\mathrm{a}, \mathrm{b}, \mathrm{c}}$ Means within the same row without a common superscript differ significantly $(\mathrm{P}<0.05) . \mathrm{n}=4$.

$\mathrm{SO}=$ Soybean oil; $\mathrm{CFP}=$ Commercial fish product; $\mathrm{CO}=$ Canola oil; $\mathrm{FCO}=50 \%$ Fish oil $+50 \%$ Canola oil.

The meat quality such as firmness, color, flavor, tenderness and juiciness are affected by the meat fatty acids 
composition. The firmness may influence by melting point of fatty acids, for instance, the melting point are $69.6{ }^{\circ} \mathrm{C}, 13.4{ }^{\circ} \mathrm{C},-5{ }^{\circ} \mathrm{C}$ and $-11{ }^{\circ} \mathrm{C}$ in $\mathrm{C} 18: 0, \mathrm{C} 18: 1, \mathrm{C} 18: 2$ and $\mathrm{C} 18: 3$, respectively, thus, the more PUFA, the less firmness (Enser, 1984). The tenderness and juiciness are concern with the amount of fatty acids, since fat could hold more water inside (Enser, 1984). The meat color will be affected by oxidation of fatty acids, which will change the red oxymyoglobin to the brown metmyoglobin (Mottram, 1998), thus, the meat more PUFA, when storage the more prone to oxidation and change color. Thus, antioxidant additives, including vitamin E, polyphenols and flavonoids are often supplied with PUFA to prevent oxidation (Lien et al., unpublished). The flavor of meat is associated with volatile compounds derived from fatty acid and peroxides from oxidation process. PUFA content in phospholipids was particularly associated with meat flavor (Mottram, 1998).

The L, a, b value, water holding capacity, fat content, POV, DPPH-scavenging ability were not affected by different dietary oils (Table 9; $\mathrm{P}<0.05$ ). TBARS of SO group was higher than $\mathrm{CO}$ group after been frozen storage for 15 days indicated that it had higher degree of oxidation $(\mathrm{P}<0.05)$, but still no different with the lard group ( $\mathrm{P}>0.05)$. This result was in contrast with Bryhni et al. (2002). Which might be due to a short time of storage in this trial. Although the composition of PUFA between CO and SO diet were very similar, the TBARS of SO was higher than that of $\mathrm{CO}$ group $(\mathrm{P}<0.05)$, the reason is needed further study. This study indicated that the different oil supplementation still no affect meat oxidation as storage meat for 15 days.

The flavor, texture, juiciness and total acceptance of longissmus muscle in SO was better than CO group (Table 10; $\mathrm{P}<0.05$ ), according to the opinion of Cameron and Enser (1991), this result might be due to the higher meat SFA contain in SO group (Tables 7 and 8), but it was difficult to explain the result obtained from lard group. All parameters of sensory evaluation in belly meat did not show any difference among groups $(\mathrm{P}>0.05)$, it is consistence with the report of Lu et al. (2008). This study results indicated that feeding pigs with CEP and FCO did not significantly influence meat quality compare to control, $\mathrm{CO}$ group influent also slightly.

\section{Conclusion}

Different dietary oils did not influent the growth performances and carcass characteristics of finishing-pigs. Animal body fatty acids compositions could be influenced by different dietary oils supplementation. Saturated fatty acids would be reduced meanwhile PUFA would be increased followed CO and SO supplementation. CFP, $\mathrm{CO}$ and FCO diets could reduce meat n-6:n-3 ratio, and the effect would be much obvious as supplemental time became longer. They did not affect storage meat antioxidation traits and meat quality. FCO group could reduce plasma cholesterol, LDL and LDL-C and increase HDL and HDL-C contain, it is benefit for consumer health. Thus, feeding pigs with low n-6:n-3 ratio fat could production healthy pork for consumers.

\section{References}

Baik, I., Abbott, R. D., Curb, J. D., \& Shin, C. (2010). Intake of fish and n-3 fatty acids and future risk of metabolic syndrome. Journal of the American Dietetic Association, 110, 1014-1017. http://dx.doi.org/10.1016/j.jada.2010.04.013

Bryhni, E. A., Kjos, N. P., Ofstad, R., \& Hunt, M. (2002). Polyunsaturated fat and fish oil in diets for growing-finishing pigs: effects on fatty acid composition and meat, fat, and sausage quality. Meat Science, 62, 1-8. http://dx.doi.org/10.1016/S0309-1740(01)00211-X

Byoung, K. A., Banno, C., Xia, Z. S., Tanaka, K., \& Ohtani, S. (1997). Effect of dietary fat sources on lipid metabolism in growing chicks (Gallus domesticus). Comperative Biochemistry Physiology-Part B, 116, 119-125. http://dx.doi.org/10.1016/S0305-0491(96)00182-4

Cameron, N. D., \& Enser, M. B. (1991). Fatty acid composition of lipid in Longissimus dorsi muscle of duroc and british landrace pigs and its relationship with eating quality. Meat Science, 29, 295-307. http://dx.doi.org/10.1016/0309-1740(91)90009-F

Casós, K., Zaragozá, M. C., Zarkovic, N., Zarkovic, K., Andrisic, L., Portero-Otín, M., ... Mitjavila, M. T. (2010). A fish-oil-rich diet reduces vascular oxidative stress in apoE(-/-) mice. Free Radical Research, 44, 821-829. http://dx.doi.org/10.3109/10715762.2010.485992

Chang, C. L., Seo, T., Du, C. B., Accili, D., \& Deckelbaum, R. J. (2010). n-3 Fatty acids decrease arterial low-density lipoprotein cholesterol delivery and lipoprotein lipase levels in insulin-resistant mice.

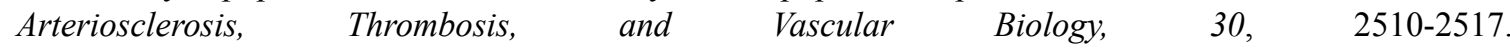
http://dx.doi.org/10.1161/ATVBAHA.110.215848

Chung, Y. C., Chang, C. T., Chao, W. W., Lin, C. F., \& Chou, S. T. (2002). Antioxidative activity and safty of the $50 \%$ ethanolic extract from red bean fermented by Bacillus subtilis IMR-NK1. Journal of Agriculture and 
Food Chemistry, 50, 2454-2458. http://dx.doi.org/10.1021/jf011369q

Department of Health. (1994). Nutritional Aspects of Cardiovascular Disease. Report on Health and Social Subject No. 46. London: Her Majesty's Stationery Office.

Dobiasova, M., Stribrna, J., Sparks, D. L., Pritchard, P. H., \& Frohlich, J. J. (1991). Cholesterol esterification rates in very low density lipoprotein- and low density lipoprotein-depleted plasma. Relation to high density lipoprotein subspecies, sex, hyperlipidemia, and coronary artery disease. Arteriosclerosis, Thrombosis, and Vascular Biology, 11, 64-70. http://dx.doi.org/10.1161/01.ATV.11.1.64

Enser, M. (1984). The chemistry, biochemistry and nutritional importance of animal fats. In J. Wiseman (Ed.), Fats in animal nutrition (pp. 23-51). London: Butterworths.

Enser, M. (2001). The role of fats in human nutrition. In B. Rossell (Ed.), Oils and fats (Vol. 2). Animal carcass fats (pp. 77-122). Leatherhead, Surrey, UK: Leatherhead Publishing.

Folch, T., Lees, M., \& Stanley, G. H. S. (1957). A simple method for the isolation and purification of total lipids from animal tissues. Journal of Biological Chemistry, 226, 499-509. http://dx.doi.org/10.2739/kurumemedj.10.12

Fraga, C. G., \& Leibovitz, B. E. (1988). Tappel. Lipid peroxidation measured as thiobarbituric acid-reactive substance in tissue slices: Characrization and comparison between homogenates and microsome. Free Radical Biology and Medicine, 4, 155-161. http://dx.doi.org/10.1016/0891-5849(88)90023-8

García-Rebollar, P., Cachaldora, P., Alvarez, C., Blas, C. D., \& Méndez, J. (2008). Effect of the combined supplementation of diets with increasing levels of fish and linseed oils on yolk fat composition and sensorial quality of eggs in laying hens. Animal Feed Science and Technology, 140, 337-348. http://dx.doi.org/10.1016/j.anifeedsci.2007.03.006

Jiang, Z., \& Sim, J. S. (1992). Effects of dietary n-3 fatty acid enriched chicken eggs on plasma and tissue cholesterol and fatty acid composition of rats. Lipids, 27, 279-284. http://dx.doi.org/10.1007/BF02536476

Kouba, M., Benatmane, F., Blochet, J. E., \& Mourot, J. (2008). Effect of a linseed diet on lipid oxidation, fatty acid composition of muscle, perirenal fat, and raw and cooked rabbit meat. Meat Science, 80, 829-834. http://dx.doi.org/10.1016/j.meatsci.2008.03.029

Lesiak, M. T., Olson, D. G., Lesiak, C. A., \& Ahn, D. U. (1997). Effects of post-mortem time before chilling and chilling temperatures on water-holding capacity and texture of turkey breast muscle. Poultry Science, 76, 552-556. http://dx.doi.org/10.1093/ps/76.3.552

Lien, T. F., Wu, C. P., \& Lu, J. J. (2003). Effect of cod liver oil and chromium picolinate supplements on the serum traits, egg yolk fatty acid and cholesterol content in laying hen. Asian-Australasian Journal of Animal Science, 16, 1177-1181. http://dx.doi.org/10.5713/ajas.2003.1177

Lu, P., Zhang, L. Y., Yin, J. D., Everts, A. K. R., \& Li, D. F. (2008). Effects of soybean oil and linseed oil on fatty acid compositions of muscle lipids and cooked pork flavor. Meat Science, 80, 910-918. http://dx.doi.org/10.1016/j.meatsci.2008.04.010

Mitchaothai, J., Yuangklang, C., Wittayakun, S., Vasupen, K., Wongsutthavas, S., Srenanul, P., ... Beynen, A. C. (2007). Effect of dietary fat type on meat quality and fatty acid composition of various tissues in growing-finishing swine. Meat Science, 76, 95-101. http://dx.doi.org/10.1016/j.meatsci.2006.10.017

Mottram, D. S. (1998). Flavour formation in meat and meat products: a review. Food Chemistry, 62, 415-424. http://dx.doi.org/10.1016/S0308-8146(98)00076-4

National Research Council. (1998). Nutrient requirements of swine (10th ed.). National Academy Press. Washington, D. C.

Oh, S. Y., Lin, C. H. H., Ryue, J., \& Brown, D. (1994). Eggs enriched with omega-3 fatty acid as a wholesome food. Journal of Apply Nutrition, 46, 15-25. http://dx.doi.org/10.1093/ps/81.12.1873

Osawa, T., Katsuzaki, H., Hagiwara, Y., Hagiwara, H., \& Shibamoto, T. (1992). A novel antioxidant isolated from young green barley leave. Journal of Agriculture and Food Chemistry, 40, 1135-1140. http://dx.doi.org/10.1021/jf00019a009

Prabhakara, R. P. G., Jyothirmayi, T., Karuna, M. S. L., \& Prasad, R. B. N. (2010). Studies on lipid profile and fatty acid composition of Roe from Rohu (Labeo rohita) and Murrel (Channa striatus). Journal of Oleo Science, 59, 515-519. http://dx.doi.org/10.5650/jos.59.515 
Riediger, N. D., Othman, R., Fitz, E., Pierce, G. N., Suh, M., \& Moghadasian, M. H. (2008). Low n-6:n-3 fatty acid ratio, with fish- or flaxseed oil, in a high fat diet improves plasma lipids and beneficially alters tissue fatty acid composition in mice. European Journal of Nutrition, 47, 153-160. http://dx.doi.org/10.1007/s00394-008-0709-8

Sebranek, J. G. (1978). Chapter 14: Oxidation measurements for fats. Meat Science and Processing (p. 132). Printed in USA.

Shackelford, S. D., Reagan, J. O., Haydon, K. D., \& Miller, M. F. (1990). Effects of feeding elevated levels of monounsaturated fats to growing-finishing swine on acceptability of boneless hams. Journal of Food Science, 55, 1485-1517. http://dx.doi.org/10.1111/j.1365-2621.1990.tb03549.x

Simopoulos, A. P., \& Salem, N. Jr. (1992). Egg yolk as a source of long-chain polyunsaturated fatty acid in infant feeding. American Journal of Clinical Nutrition, 55, 411-414. http://dx.doi.org/10.3945/ajcn.2010.29495

Statistical Analysis System Institute Inc. (1998). SAS/STAT User's guide: statistics (Version 6.06). Cary, NC, USA: SAS Institute Inc.

Tai, C. C., \& Ding, S. T. (2010). N-3 polyunsaturated fatty acids regulate lipid metabolism through several inflammation mediators: mechanisms and implications for obesity prevention. The Journal of Nutritional Biochemistry, 21, 357-363. http://dx.doi.org/10.1016/j.jnutbio.2009.09.010

Teye, G. A., Sheard, P. R., Whittington, F. M., Nute, G. R., Stewart, A., \& Wood, J. D. (2006). Influence of dietary oils and protein level on pork quality. 1. Effects on muscle fatty acid composition, carcass, meat and eating quality. Meat Science, 73, 157-165. http://dx.doi.org/10.1016/j.meatsci.2005.11.011

Thacker, P. A. (1998). Effect of micronization of full-fat canola seed on performance and carcass characteristics of growing-finishing pigs. Animal Feed Science and Technology, 71, 89-97. http://dx.doi.org/10.1016/S0377-8401(97)00133-8

Wan, J. B., Huang, L. L., Rong, R., Tan, R., Wang, J., \& Kang, J. X. (2010). Endogenously decreasing tissue n-6/n-3 fatty acid ratio reduces atherosclerotic lesions in apolipoprotein E-deficient mice by inhibiting systemic and vascular inflammation. Arteriosclerosis, Thrombosis, and Vascular Biology, 30, 2487-2494. http://dx.doi.org/10.1161/ATVBAHA.110.210054

Warnants, N., Van, Oeckel, M. J., \& Boucqué, C. V. (1999). Incorporation of dietary polyunsaturated fatty acids into pork fatty tissues. Journal of Animal Science, 77, 2478-2490. http://dx.doi.org/10.1016/S0309-1740(98)00011-4

West, R. L., \& Myer, O. L. (1987). Carcass and meat quality characteristics and backfat fatty acid composition of swine as affected by the consumption of peanuts remaining in the field after harvest. Journal of Animal Science, 65, 475-480. http://dx.doi.org/10.4141/cjas93-053

Wood, J. D., Nute, G. R., Richardson, R. I., Whittington, F. M., Southwood, O., Plastow, G., ... Chang, K. C. (2004). Effects of breed, diet and muscle on fat deposition and eating quality in pigs. Meat Science, 67, 651-667. http://dx.doi.org/10.1016/j.meatsci.2004.01.007

Wood, J. D., Richardson, R. I., Nute, G. R., Fisher, A. V., Campo, M. M., Kasapidou, E., ... Enser, M. (2003). Effects of fatty acids on meat quality: A review. Meat Science, 66, 21-32. http://dx.doi.org/10.1016/S0309-1740(03)00022-6

\section{Copyrights}

Copyright for this article is retained by the author(s), with first publication rights granted to the journal.

This is an open-access article distributed under the terms and conditions of the Creative Commons Attribution license (http://creativecommons.org/licenses/by/3.0/). 